\title{
Inter-DNA Electrostatics from Explicit Solvent Molecular Dynamics Simulations
}

\author{
Alexey Savelyev and Garegin A. Papoian* \\ Department of Chemistry, University of North Carolina at Chapel Hill, Chapel Hill, North Carolina 27599-3290 \\ Received January 10, 2007; E-mail: gpapoian@unc.edu
}

Electrostatic interactions drive a compaction of many biomolecules, such as RNA and DNA. ${ }^{1,2}$ For example, DNA is compacted a million-fold into a highly organized structure in eukaryotic cells, called chromatin. ${ }^{2-5}$ Understanding the mechanism of chromatin folding is of great biological importance, since it mediates the extent of accessibility of specific DNA sequences, which in turn, controls important DNA-templated processes, such as gene expression, recombination, and repair. ${ }^{3}$ Although the structure of chromatin has not yet been determined, a number of structural models have been put forward. ${ }^{4,5}$ All-atom molecular dynamics (MD) simulations could provide crucial insights into the electrostatic and structural mechanisms of chromatin folding. Because of the enormous size of even a short chromatin fiber segment and long folding timescales, all-atom MD simulations of this process will remain computationally impractical in the foreseeable future. A number of alternative coarse-grained (CG) models of the chromatin fiber have been developed. ${ }^{6-8}$ However, these models treat electrostatics of the whole nucleosome, the basic repeating unit of chromatin, by either approximate Poisson-Boltzmann (PB) theory or explicit treatment of the ions, utilizing a continuum dielectric approximation for the aqueous solvent with drastically simplified representations of the polynucleosomal structure. ${ }^{6-8}$

Our recent all-atom MD simulations of a 16 base pair DNA oligomer, solvated in various $\mathrm{NaCl} / \mathrm{KCl}$ buffers, pointed to substantial differences between the more exact MD simulations and the predictions from the PB theory at distances less than $10 \AA$ from the DNA surface. ${ }^{9}$ Consequently, the standard models of continuum electrostatics ${ }^{10}$ are not fully adequate when surfaces of two linker DNA chains, connecting adjacent nucleosomes, are less than $20 \AA$ apart. In addition, we observed qualitatively different $\mathrm{Na}^{+}$and $\mathrm{K}$ + condensation patterns around DNA, resulting in dissimilar conformational dynamics of DNA segment in $\mathrm{NaCl}$ and $\mathrm{KCl}$ buffers. ${ }^{9}$ This suggests that ion-specific modeling is required to describe electrostatics at short distances, while the ionic differences have been ignored in the PB approach.

Our long-term aim is to build an accurate CG model of the chromatin, derived systematically from all-atom simulations. As a first step toward achieving this goal, we report here the free energy profiles for bringing to proximity two in-parallel oriented 16 base pair DNA segments in $\mathrm{NaCl}$ and $\mathrm{KCl}$ salt buffers (Figure 1), computed from a series of extensive all-atom MD simulations in explicit solvent using AMBER 8.0 (see the Supporting Information for system preparation details, the MD simulation protocol, and the free-energy calculation details). The total simulation time was $480 \mathrm{~ns}$ for systems containing 43000 atoms, making these DNA simulations among the largest reported to date. On the basis of these results, we have developed salt-specific, short-range effective interaction potentials between two CG linker DNA chains, that will serve as a key component for the polynucleosomal array CG forcefield.

We have found that when two DNA oligomers are brought into proximity (see Figure 1), the resulting repulsion profile is steeper
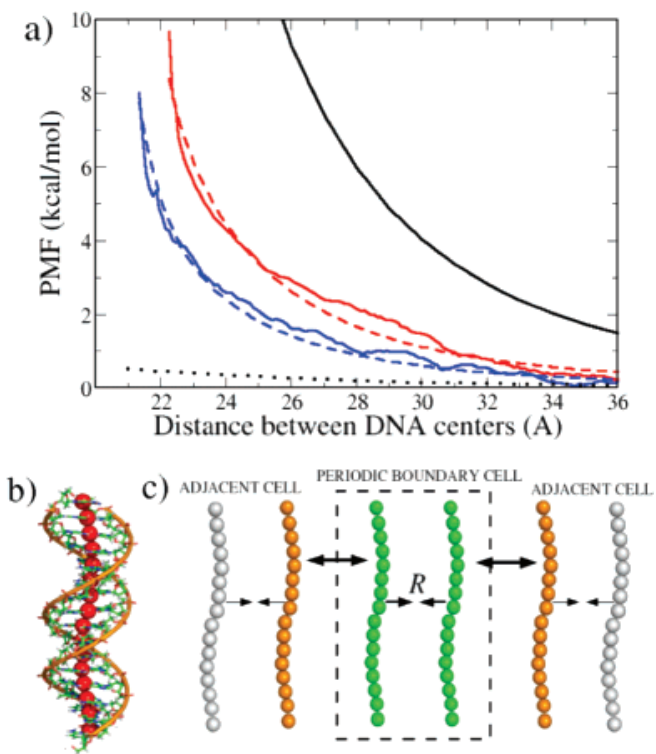

Figure 1. (a) Potentials of mean force (PMF) for bringing to proximity two 16 base pair DNA oligomers, in parallel orientation, in aqueous solution with $\mathrm{NaCl}$ (blue) and $\mathrm{KCl}$ (red) salt buffers. PMFs were calculated from a series of extensive all-atom MD simulations using the AMBER force-field (see the Supporting Information). The dotted and solid black lines indicate the electrostatic energy of CG DNAs, interacting via conventional and modified Debye-Hückel (DH) potentials, respectively, the latter accounting for DNA size. ${ }^{10}$ The modified potential was subsequently rescaled by fitting to the MD simulation results, resulting in the blue and red dashed curves, for the $\mathrm{NaCl}$ and $\mathrm{KCl}$ buffers, respectively (see the text and panel c. (b) CG DNA is modeled as a sequence of beads (red spheres), where a single bead is placed in each base pair geometric center. (c) We calculated electrostatic energy of two CG DNAs in periodic boundary cell (green), interacting via above-mentioned potentials, as a function of center-to-center distance $R$. To make an appropriate comparison with all-atom simulation results, the interactions with the nearest neighbor DNAs from the adjacent periodic cells (orange) were taken into account.

in the $\mathrm{KCl}$ buffer compared with $\mathrm{NaCl}$. Since increased counterion condensation around DNA is expected to facilitate inter-DNA aggregation, the obtained free-energy profiles indicate that $\mathrm{Na}^{+}$ screens DNA more efficiently than $\mathrm{K}^{+}$. This conclusion is in agreement with the prior experiments on the compaction of a long DNA chain facilitated by various monocations. ${ }^{11}$ More recent experimental work, reported by the same group, has demonstrated that $\mathrm{Na}^{+}$uncompacts DNA, previously compacted by spermidine $(3+)$, significantly more efficiently than $\mathrm{K}^{+} .12$ This uncompaction process may be naturally viewed as binding competition for DNA between the monovalent ions, at very high abundance, and spermidine $(3+)$. Consequently, these results suggest that the $\mathrm{Na}^{+}$ ions bind stronger to DNA compared with the $\mathrm{K}^{+}$ions, in agreement with our findings. In an unrelated set of experiments, Parsegian group reported direct measurements of forces between doublestranded DNA chains in ordered array solutions of various halides of monovalent cations. ${ }^{13}$ These experiments revealed that the 


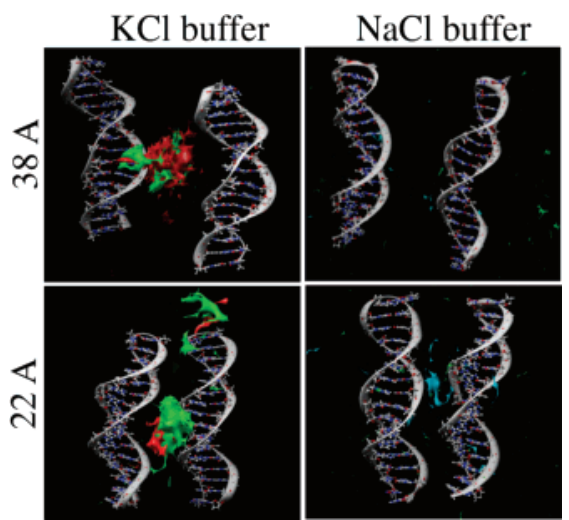

Figure 2. Distributions of $\mathrm{Na}^{+}$(blue), $\mathrm{K}^{+}$(red), and $\mathrm{Cl}^{-}$(green) ions around the average DNA structures, obtained by superposition of all snapshots of $\mathrm{MD}$ simulations in $\mathrm{KCl}$ (left) and $\mathrm{NaCl}$ (right) buffers. The shown ionic clouds are isosurfaces corresponding to $>80 \%$ of the maximum ion density. Distributions are shown for two center-to-center distances, at which DNA segments were held: at the largest ( $38 \AA$ ), and smallest ( $22 \AA$ ) separations.

interchain repulsion forces, measured as a function of distance between DNA segments, noticeably increased when $\mathrm{NaCl}$ buffer was replaced by $\mathrm{KCl} .{ }^{13}$ Hence, our computed PMFs in two buffers are in substantial agreement with these experimental findings.

We have recently described several mechanisms rationalizing the higher binding affinity to DNA for $\mathrm{Na}^{+}$, compared with $\mathrm{K}^{+} .9$ In particular, we have observed the formation of $\mathrm{K}^{+} \mathrm{Cl}^{-}$clusters, resulting in efficient $\mathrm{K}^{+}$screening. Similar $\mathrm{Na}^{+} \mathrm{Cl}^{-}$clusters do not form under these conditions. ${ }^{9}$ The $\mathrm{K}^{+} \mathrm{Cl}^{-}$clusterization may also play an important role in determining the difference in the interDNA free-energy profiles, obtained in this work, reducing electrostatic screening of the DNA-DNA repulsion. Additionally, these clusters provide steric interference for two approaching DNA chains. To illustrate this point, we have built the $\mathrm{Na}^{+}, \mathrm{K}^{+}$, and $\mathrm{Cl}^{-}$charge isosurfaces, corresponding to $>80 \%$ of the maximum ion density, for three different distances between DNA oligomers (Figure 2). It is seen that dissimilar clusterization leads to qualitatively different charge distributions in $\mathrm{NaCl}$ and $\mathrm{KCl}$ buffers. In the former case, $\mathrm{Na}^{+}$and $\mathrm{Cl}^{-}$are spread more uniformly across the system, as indicated by the absence of significant high-density regions. On the other hand, high spatial co-localization of $\mathrm{Cl}^{-}$and $\mathrm{K}^{+}$is a striking indication of $\mathrm{K}^{+} \mathrm{Cl}^{-}$clusterization. As two DNAs approach each other, the $\mathrm{K}^{+} / \mathrm{Cl}^{-}$high-density region becomes segregated, indicating a cluster splitting (see Figure 2).

To verify the force-field specificity of the obtained higher $\mathrm{Na}^{+}$ condensation on DNA, we carried out simulations of the same DNA oligomer in $\mathrm{NaCl}$ and $\mathrm{KCl}$ salt buffers, using the CHARMM forcefield, which will be published elsewhere. It turned out that, although the $\mathrm{K}^{+} \mathrm{Cl}^{-}$clustering is much less pronounced in CHARMM, $\mathrm{Na}^{+}$ ions are still condensed around DNA to a larger degree compared to $\mathrm{K}^{+}$, albeit with reduced specificity (by $\sim 5 \%$ within $9 \AA$ from the DNA surface, compared with $\sim 20 \%$ in AMBER simulations). This indicates that $\mathrm{K}^{+} \mathrm{Cl}-$ clustering, which is significant in AMBER, is just one of several mechanisms determining the relative extent of $\mathrm{Na}^{+}$and $\mathrm{K}^{+}$binding to DNA. Since both force-fields were designed to reproduce the available experimental data on $\mathrm{NaCl}$ and $\mathrm{KCl}$ electrolyte solutions, the incomplete agreement between AMBER and CHARMM results reflects the lack of the broader experimental knowledge about the structural and energetic properties of ionic aqueous solutions. ${ }^{14}$ Comparisons of our computational results with the experimental electrolyte activity curves suggest that the clustering behavior of a dilute $\mathrm{KCl}$ solution might be intermediate between the AMBER and CHARMM predictions, which will be discussed elsewhere.
We discuss next the procedure for obtaining effective short-range interaction potentials between two CG DNAs from the all-atom PMF curves in Figure 1a. A model of CG DNA chain is depicted in Figure $1 \mathrm{~b}$. The long-range interactions were described with the Debye-Hückel (DH) potential, where the effective bead charge is taken to be approximately a quarter of the bare DNA charge. ${ }^{15}$ This assumption allowed us to set the absolute scale of the inter-DNA free energy curve (Figure 1), by equating the free energy for two DNAs at the largest separation in our all-atom simulations to the interaction energy calculated from the analytical DH potential (see the dotted line in Figure 1a). Note that at shorter distances the conventional $\mathrm{DH}$ potential significantly underestimates the interaction energy (compare the black dotted line with solid red and blue curves). Next, using the value of $q_{\text {eff }}=-0.5$ for bead charge ${ }^{15}$ and introducing an interbead potential, $e^{-\kappa(r-a)} /(r-a)$, which is modified from the DH potential for a spherical ion of radius $a,{ }^{10}$ one arrives at the interaction energy between two CG DNAs depicted as a solid black line in the Figure 1a. We used $\kappa^{-1}=9 \AA$, which is the Debye length at physiological conditions, and $a=20$ $\AA$, which is the double-stranded DNA diameter, the latter accounting for the difference between 3D atomistic DNA structure and the pointlike bead CG model. To match this energy with the potentials of mean force for $\mathrm{NaCl}$ and $\mathrm{KCl}$ salt buffers, we scaled the above potentials by the constants $\phi_{0}^{\mathrm{Na}^{+}}=0.15$ and $\phi_{0}^{\mathrm{K}^{+}}=0.28$, respectively. The resulting short-range effective potentials, representing the energy of DNA-DNA electrostatic repulsion, reproduce in a very efficient way the all-atom MD simulation results in two buffers (see Figure 1a).

In the forthcoming work, the effective bead charge will be derived to reproduce the DNA long-range electrostatic potential, obtained from all-atom MD simulations of a single DNA oligomer, ${ }^{9}$ instead of relying on the Manning's counterion condensation theory. ${ }^{15}$ We expect this to result in small adjustments to the scaling constants, $\phi_{0}^{\mathrm{Na}^{+}}$and $\phi_{0}^{\mathrm{K}^{+}}$.

Acknowledgment. We are grateful to Dr. Michael Rubinstein, Dr. Kenichi Yoshikawa, and Dr. Anatoly Zinchenko for helpful discussions. This work was supported through North Carolina Biotechnology Center Grant 2006-MGR-1107. All calculations were carried out using the UNC Topsail supercomputer.

Supporting Information Available: Details on system preparation and parametrization and the description of the all-atom MD simulation protocol. This material is available free of charge via the Internet at http://pubs.acs.org.

\section{References}

(1) Kocli, E.; Hyeon, C.; Thurmalai, D.; Woodson, S. A. J. Am. Chem. Soc 2007, 129, 2676-2682.

(2) Schiessel, H. J. Phys.: Condens. Matter 2003, 15, R699-R774.

(3) Luger, K.; Hansen, J. C. Curr. Opin. Struct. Biol. 2005, 15, 188-196.

(4) Dorigo, B.; Schalch, T.; Kulangara, A.; Duda, S.; Schroeder, R. R Richmond, T. J. Science 2004, 306, 1571-1573.

(5) Schalch, T.; Duda, S.; Sargent, D. F.; Richmond, T. J. Nature 2005, 436, $138-141$.

(6) Arya, G.; Zhang, Q.; Schlick, T. Biophys. J. 2006, 91, 133-150.

(7) Korolev, N.; Lyubartsev, A. P.; Nordenskild, L. Biophys. J. 2006, 90 4305-4316.

(8) Langowski, J. Eur. Phys. J. E 2006, 19, 241-249.

(9) Savelyev, A.; Papoian, G. A. J. Am. Chem. Soc. 2006, 128, 14506-14518.

(10) Evans, D. F.; Wennerstrom, H. The Colloidal Domain Where Physics, Chemistry, Biology, and Technology Meet; Wiley-VCH: New York, 1999.

(11) Zinchenko, A. A.; Yoshikawa, K. Biophys. J. 2005, 88, 4118-4123.

(12) Hibino, K.; Yoshikawa, Y.; Murata, S.; Saito, T.; Zinchenko, A. A Yoshikawa, K. Chem. Phys. Lett. 2006, 426, 405-409.

(13) Podgornik, R.; Rau, D. C.; Parsegian, V. A. Biophys. J. 1994, 66, 962971.

(14) Patra, M.; Karttunen, M. J. Comput. Chem. 2004, 25, 678-689.

(15) Manning, G. S. J. Chem. Phys. 1969, 51, 924-933.

JA070207T 